\title{
La telesecundaria y el telebachillerato comunitario en cuestión. Una mirada desde los maestros rurales de Tierras Negras*
}

\author{
Álvaro Andrés Rivera Sepúlveda \\ María Alicia Zavala Berbena ${ }^{2}$
}

Recibido: 05-05-2020

Aceptado: 16-06-2020

\section{Resumen}

La investigación que enmarca el presente artículo indagó, desde la óptica de los maestros de una localidad del Estado de Guanajuato, por el funcionamiento de dos modelos educativos mexicanos que operan en el medio rural: la telesecundaria y el telebachillerato comunitario. Metodológicamente se diseñó un estudio cualitativo haciendo uso del grupo de discusión como técnica central para el diálogo con los maestros in situ. Los resultados dejan entrever las fisuras que presentan los dos modelos en orden a ofrecer una educación de calidad para la población rural, entre otras: el rezago académico de los estudiantes producto de la educación recibida en los niveles precedentes y las dificultades que esto supone para

\footnotetext{
*Artículo de investigación derivado del proyecto Eficiencia en la cobertura y calidad de la educación. Caso El Polígono de Tierras Negras, León-México, financiado por el Sistema de Redes de Investigación Aplicada. Año 2019

1. PhD. en Educación. Docente investigador de carrera, Universidad Militar Nueva Granada, Bogotá, Colombia.

Correo electrónico: alvaro.rivera@unimilitar.edu.co

ORCID: https://orcid.org/0000-0002-7405-7988

Google scholar: https://scholar.google.com/citations?hl=es\&user=_8V4qVIAAAAJ

2. PhD. en Educación. Directora de Investigación de la Universidad De La Salle Bajío, León, México.

Correo electrónico: azavala@delasalle.edu.mx

ORCID: https://orcid.org/ 0000-0002-9954-4539
} 
el despliegue de los principios pedagógicos de los dos modelos, asimismo, la discontinuidad que se presenta entre la formación permanente que reciben los maestros y las dificultades reales que éstos encaran en la cotidianidad de la vida escolar.

Palabras clave: Educación rural, televisión educativa, educación básica, programas de educación, docente de secundaria.

\section{Community's telesecundaria and telebachillerato in question A view from the rural masters of Tierras Negras.}

\section{Abstract}

The research framed in this article was investigated from the perspective of teachers from a locality in the State of Guanajuato, explains the functioning of two mexican educational models operating in rural areas: telesecundaria and telebachillerato. Methodologically, a qualitative study using discussion groups as a central technique for dialogue with the teachers in situ, was designed. The results showed fissures presented by the two models, in order to provide quality education for the rural population, among others: the academic lay back of students is the result from the education received at the previous levels and the difficulties, this entails the deployment of the pedagogical principles of the two models. In addition, there is a discontinuity between the continuous training received by the teachers and the real difficulties they face in everyday scholastic life.

Keywords: Rural education, educational television, elementary and middle education, education programs, middle teacher. 


\section{Introducción}

El modelo de educación telesecundaria nació en México en 1968 con el propósito de implementar educación secundaria utilizando la transmisión televisiva como base del proceso pedagógico. Con esta medida se pretendió dar respuesta a la demanda creciente por estudiar este nivel educativo aprovechando los avances de las tecnologías de la información y la comunicación, y de este modo satisfacer el derecho a la educación para jóvenes de medios rurales y urbano marginales (Jiménez, Martínez., \& García, 2010). Desde su entrada en vigor, el impacto de la telesecundaria en el aumento de la matrícula fue inmediato: de 6569 alumnos inscritos en 1968, se ascendió a 23762 en sólo dos ciclos escolares (Calixto \& Rebollar, 2008)

En 2003 se realizó una revisión de la efectividad del sistema con base en los logros de aprendizaje obtenidos por los estudiantes en dos pruebas: ENLACE (Evaluación Nacional de Logros Académico en Centros Escolares) y PISA (Programme for International Student Assessment) (Jiménez, Martínez, \& García, 2010). En líneas generales, los resultados demostraron que los aprendizajes de los estudiantes se situaban por debajo de lo esperado; al hacer un análisis de la operación, organización y recursos con los que cuenta la telesecundaria en zonas rurales y marginales de México, se concluyó que estos centros educativos reproducen las inequidades sociales iniciales o de punto de partida (Santos del Real y Carbajal, 2001).

En 2011 se formuló un modelo educativo para las telesecundarias que, si bien destacaba el uso de la televisión como principal apoyo tecnológico, confirió mayor protagonismo al docente de la sede como responsable de los procesos de enseñanza-aprendizaje, además, incorporó recursos pedagógicos, formación docente, infraestructura y equipamiento (Ver Figura 1). 
Figura 1. Modelo Educativo para el Fortalecimiento de la Telesecundaria en México.

\section{Servicio Educativo de Telesecundaria}

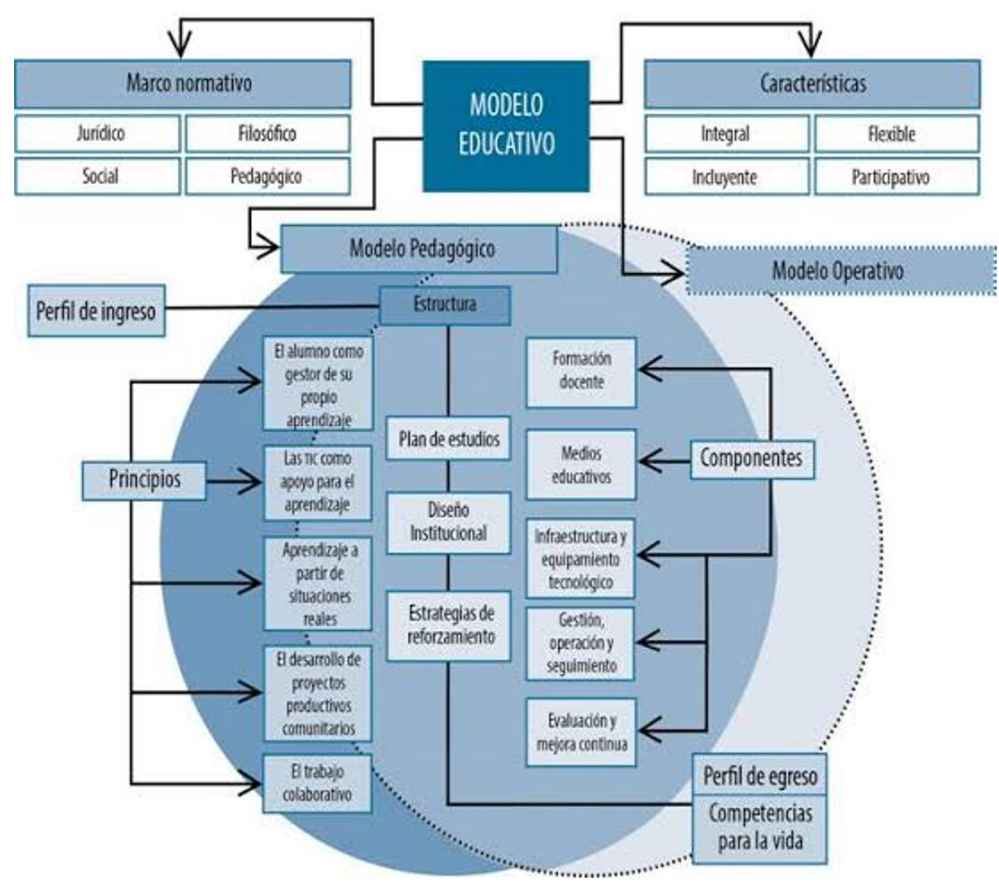

Fuente: Secretaria de Educación Pública (2011).

Actualmente en México operan 18720 escuelas telesecundarias públicas, de las cuales el 88.5 por ciento se ubican en comunidades rurales y casi el 90 por ciento brindan sus servicios en localidades de alto y muy alto grado de marginación social. No obstante, los resultados de PLANEA-ELSEN (Plan Nacional de Evaluación de los Aprendizajes en su modalidad de logro, referida al Sistema Educativo Nacional) 2017, han evidenciado que los alumnos de telesecundarias logran menores grados de aprendizaje en comparación con otras modalidades de servicio en este mismo nivel. El 48.8 por ciento de los alumnos obtuvieron un grado de logro insuficiente, específicamente en el dominio de lenguaje y comunicación (INEE, 
2019). Lo anterior permite concluir que, después de 51 años de funcionamiento, la telesecundaria se ha constituido como una alternativa a las necesidades educativas en México, sin embargo, presenta deficiencias significativas que requieren ser caracterizadas y atendidas.

Por otra parte, el aumento de los egresados de la telesecundaria provocó un fenómeno por presión desde abajo que condujo de igual manera al aumento de la demanda por la educación media superior, lo que desembocó en la década de los ochentas en la creación de telebachilleratos de modalidad escolarizada, cuyo propósito consistió en ampliar la cobertura, aliviar el rezago educativo y fomentar el arraigo de los jóvenes en sus comunidades de origen, especialmente en localidades dispersas en las que no era factible la construcción de un centro educativo completo, siendo el estado de Veracruz uno de sus precursores (Salazar, 2007).

El telebachillerato comunitario (TBC) es la adaptación de este programa a los contextos rurales y urbano marginales de hasta 2500 habitantes, su pretensión es ofrecer educación media superior a los jóvenes egresados de la telesecundaria. En la mayoría de los estados, los TBC funcionan a contramuro en los planteles de la secundaria o en espacios que la misma comunidad dispone para la prestación del servicio; operan bajo la modalidad escolarizada presencial y cuenta con la presencia permanente de tres docentes, uno para el área de matemáticas y ciencias experimentales, otro para las ciencias sociales y humanidades y el último para comunicación. Los procesos de enseñanza-aprendizaje se orientan por medio de libros de texto gratuitos diseñados exclusivamente para el programa, cuadernos de ejercicios y clases grabadas en video.

Para abrir un TBC se realiza un estudio de factibilidad que consiste en identificar que no exista otro servicio de este nivel en cinco kilómetros, que el tamaño de la localidad sea menor a 2500 habitantes y que se cuente con un mínimo de 12 estudiantes. Los principales usuarios de este servicio son egresados de las secundarias y telesecundarias de las mismas localidades rurales. Al momento de su 
establecimiento, el gobierno federal les asigna una computadora, un cañón y una impresora, durante el primer año también se encarga del pago de tres docentes, al segundo año la gestión estatal debe asumir el $50 \%$ de dicho pago y de los costos de operación (Guzmán, 2018: 51)

En Guanajuato, el telebachillerato comunitario es sostenido y ofrecido por el Sistema Avanzado de Bachillerato y Educación Superior (SABES), cuyos centros educativos se ubican principalmente en las zonas rurales del Estado. En su fundamentación, el TBC es concebido como un modelo que enfatiza la capacitación para el trabajo y el desarrollo de habilidades de emprendimiento (SABES, 2019). Desde su creación en 1996, el número de TBC en Guanajuato ha crecido sustancialmente, a esto ha contribuido, por una parte, el aumento de los egresados de la telesecundaria y, por otra, la declaración en 2012 del carácter obligatorio del nivel medio superior en México.

La presente investigación se desarrolló en el Polígono de Tierras Negras, una constelación de pequeñas localidades rurales dedicadas principalmente a la agricultura y a la ganadería de usufructo doméstico, situadas en la zona centro-occidente de México, estado de Guanajuato, sierra de Pénjamo. Se trata de una región elevada con barrancas y cerros altos, que dificultan los desplazamientos entre sus habitantes. El epicentro del polígono es la localidad, también Ilamada Tierras Negras, fundada en 1790, la cual a la fecha cuenta con una población de sólo 200 habitantes, misma cantidad que se conserva desde hace más de cien años debido al alto grado migratorio que la caracteriza.

En general, entre los hogares prevalece un nivel de marginación y rezago social muy alto, por ejemplo, se destaca que el 98 por ciento de las viviendas carece del servicio de agua potable (SEDESOL, 2013). El nivel de escolaridad de los pobladores es deficiente, pues mientras que la media nacional se ubica en 8.0 años de escolarización, en el Polígono es de 4.7 años escasamente. En esta zona operan dos centros educativos, uno de telesecundaria, al que asisten jóvenes entre los 12 y 15 años, y otro de telebachillerato, con 
jóvenes entre los 15 y 18 años. La población atendida pertenece a las comunidades circundantes, algunas de las cuales se encuentran a más de 10 kilómetros de distancia del complejo educativo.

Los indicadores oficiales evidencian que el abandono promedio en el nivel de secundaria es del 8 por ciento, mientras que en el nivel de bachillerato es del 25 por ciento. Asimismo, para evaluar el logro educativo en estos niveles se aplicó el PLANEA, una prueba estandarizada que evalúa aprendizajes clave del currículo escolar en las áreas de lenguaje y comunicación y matemáticas. Los resultados de estas pruebas se jerarquizan en 4 niveles: 1 = insuficiente; 2 = indispensable; 3 = satisfactorio; y 4 = sobresaliente. De acuerdo con los datos de la última aplicación (año 2017), en los centros educativos de Tierras Negras el 80 por ciento de los alumnos de secundaria y el 69 por ciento del bachillerato se ubicaron en los niveles 1 y 2 en el área de lenguaje y comunicación, asimismo, el 40 por ciento en secundaria y el 92 por ciento en bachillerato, se ubicaron en estos mismos dos niveles en el área de matemáticas, todo lo cual deja entrever que existen deficiencia importantes en los dos modelos que requieren ser atendidas.

Sobre la base de lo expuesto, y cómo una manera de trascender la visión monolítica que ofrecen las cifras oficiales, la presenté investigación indagó por el funcionamiento de la telesecundaria y el telebachillerato comunitario en el Polígono de Tierras Negras, recuperando la perspectiva que, sobre este aspecto, poseen los maestros que laboran en este sector. Se pretendía con esto reconocer, desde la óptica de sus protagonistas, las problemáticas que presentan estos modelos más allá de las idealizaciones teóricas y, por esta vía, vislumbrar alternativas para el diseño de un plan de acción por parte de las autoridades educativas del Estado de Guanajuato.

\section{Metodología}

Se llevó a cabo una indagación de corte cualitativo (subtendida desde la corriente interpretativa), por medio de grupos de discusión, orientada a la reconstrucción de las percepciones intersubjetivas 
de sentido común que ostentan un colectivo de maestros rurales sobre el funcionamiento de los dos modelos en su lugar de trabajo. La fundamentación epistemológica y metodológica del diseño se inspiró en los planteamientos ofrecidos por uno de los precursores de esta técnica, el sociólogo español Jesús Ibáñez Alonso.

La selección de los maestros para la conformación de los grupos (ver tabla 1) se adelantó siguiendo tres criterios específicos: a. la elección de una muestra estructural comprensiva: maestros que por sus características (más que por su extensión) conformaban un subconjunto pertinente para la producción de relaciones relevantes; b. composición de grupos heterogéneos incluyentes: maestros adscritos a distintos programas y con diferencias en su formación de base y pedagógica, pero que aun así no constituían ninguna relación de exclusión preexistente; c. aseguramiento de continuidad entre la macro y la microsituación: maestros para quienes el tema de discusión no les era desconocido por hacer parte también de sus conversaciones espontáneas en la vida cotidiana.

Tabla 1. Maestros participantes

\begin{tabular}{|l|l|l|l|}
\hline & \multicolumn{1}{|c|}{ Programa } & \multicolumn{1}{|c|}{$\begin{array}{c}\text { Formación } \\
\text { de base }\end{array}$} & \multicolumn{1}{|c|}{$\begin{array}{c}\text { Formación } \\
\text { pedagógica }\end{array}$} \\
\hline Maestro 1 & Telesecundaria & Ingeniero & no \\
\hline Maestro 2 & Telesecundaria & Ingeniero & sí \\
\hline Maestro 3 & Telesecundaria & Licenciado & sí \\
\hline Maestro 4 & Telebachillerato & Ingeniero & sí \\
\hline Maestro 5 & Telebachillerato & Licenciado & no \\
\hline Maestro 6 & Telebachillerato & Ingeniero & no \\
\hline
\end{tabular}

Fuente: Elaboración propia.

Con los maestros seleccionados se conformó un grupo de trabajo no necesariamente preexistente como grupo básico y mucho menos subsistente como institución. Esta conformación ad hoc se orientó principalmente a la producción de un discurso para posteriormente ser analizado. De hecho, Ibáñez sostiene que "cualquier intento de hacer, o dejar subsistir el grupo -lo que implicaría una 
institucionalización real- niega los fundamentos metodológicos de la técnica" (p. 312), ya que de esta manera el grupo podría constituirse como sujeto de una praxis y tomar la palabra, lo cual es factible desde otros pisos epistemológicos, pero no desde el que se erigió la presente investigación.

Una vez congregados, los maestros fueron motivados a discutir sobre el siguiente tópico: funcionamiento de la telesecundaria y el telebachillerato en su centro, esto se hizo compartiendo con ellos el contexto de la investigación (marco institucional y objetivos), así como haciendo hincapié en la importancia que reviste para el mismo estudio reconocer sus percepciones, experiencias y saberes. Desde esta lógica, el papel de los investigadores (preceptores), más allá de seguir un esquema de preguntas prefigurado o de irrumpir con sus propias concepciones sobre el tema, consistió en mantener viva la discusión (deshaciendo bloqueos y desatando nudos) y conservarla dentro du su campo semántico (evitando desviaciones o la focalización en asuntos subsidiarios).

Ciertamente, Ibáñez deja entrever que un grupo de discusión cumple de mejor forma su objetivo si el grupo va ganando cada vez mayor protagonismo e independencia frente al preceptor, constituyéndose de esta manera en un espacio de auténtica libertad para el intercambio de argumentos y la construcción de consensos. Aunque alcanzar este ideal no siempre es fácil, un elemento que contribuyó a ello en la presente indagación fue omitir el diseño de un formato de preguntas o subtemas prefijado, aunado a una actitud de escucha atenta e intuición permanente para conseguir (por medio de una direccionalidad moderada) que el mismo grupo fuera dilucidando los focos de discusión y el orden a seguir. Por lo tanto, lo que se presenta como resultados no corresponde a la saturación empírica de unas categorías definidas con anticipación, sino al producto consensuado de la discusión de los maestros, tanto en su contenido como en su organización.

Ahora, el análisis del discurso producido por un grupo corre de manera paralela al procedimiento de producción e involucra a todos los participantes, actuantes y preceptor. De acuerdo con 
Ibáñez, "lo que cada destinador emite como fuerza, cada destinatario lo recibe como sentido, la emisión es siempre una escritura, la recepción es siempre una lectura" (2003: 135). En lo que concierne al investigador, este actúa siempre como un 'sujeto en proceso', en permanente configuración. Esto es posible gracias a que en el decurso mismo de la discusión va desplegando facultades auténticamente humanas (intuir, imaginar, conjeturar) constituyendo microprocesos de reflexividad para la conducción del grupo. Por lo tanto, lo que el investigador registra en su grabadora y posteriormente descarga en su ordenador, es ya un texto interpretado, el cual somete a una segunda fase o nivel de análisis. Para el caso de la presente investigación, se siguió el procedimiento de conceptualización, categorización, desarrollo de categorías y redacción del informe.

\section{Resultados y discusión}

\section{Bajas expectativas educativas}

Uno de los principales problemas que se presenta en Tierras $\mathrm{Ne}$ gras está asociado a las bajas expectativas educativas que poseen las familias y los mismos estudiantes del sector. Según lo conciben los maestros, el grueso de los jóvenes desarrollan un estilo de vida surcado por la ausencia de aspiraciones y cuyo único horizonte de sentido es la reproducción de las costumbres del lugar: terminar la 'prepa', conformar un hogar a temprana edad y luchar por la sobrevivencia cotidiana; de suerte que, en su gran mayoría, son jóvenes que adolecen de un proyecto de vida autodeterminado. Así, el paso por la escuela es concebido por muchos como una etapa que se debe superar para cumplir con la tradición y no como una oportunidad de autorrealización que puede conducir a una mejor calidad de vida a nivel académico y profesional.

Lo anterior está indicando que las dificultades para operacionalizar el componente de formación para el trabajo y la realización de proyectos de desarrollo comunitario que le son propias al tele- 
bachillerato comunitario, no obedecen únicamente a la limitación de horas destinadas para ello en el plan de estudios ni al grado de complejidad que comporta esta empresa para los docentes (Weiss et al., 2017), sino también al escaso interés manifestado por los mismos estudiantes a propósito de este tipo de formación y la poca trascendencia que este esfuerzo representaría en su proyecto personal de vida.

No son extrañas, entonces, las permanentes manifestaciones de desidia y desmotivación entre los jóvenes, aun así, son enviados por sus familias a la escuela ¿por qué? Escudriñando en este aspecto los maestros manifestaron que el programa de becas ha conducido a que muchos padres de familia interpreten la permanencia de sus hijos en la escuela como una forma de solventar las necesidades del ámbito doméstico, y es por eso que muchos de ellos manifiestan interés en que el centro educativo continúe funcionando, todo lo cual ofrece un punto de vista alternativo a lo expuesto por Weiss (2017) y Guzmán (2018) cuando subrayan que el programa de becas ha contribuido a incrementar el número de jóvenes que se inscriben en la escuela. Al respecto, uno de los maestros analiza:

Sí, ya tienen muchos años, desde que yo estoy aquí, 20 años casi... a los alumnos se les dan becas; se han nombrado de diferente manera, pero es lo mismo, por estar estudiando simplemente, y la mayoría de las familias son de bajos recursos, las becas se conceden al 90-99 por ciento, es decir, casi todos tienen becas, y ese es un motivo para que las familias los manden a la escuela, y así entre más hijos tengan en la escuela más dinero tiene la familia y es una manera de subsistir.

Ahora, las subvenciones públicas han originado un nivel de dependencia profundamente arraigo entre los habitantes del sector, denominado por ellos mismos 'síndrome de la sierra', al punto que muy pocos estudiantes interpretan su proceso de escolarización como una forma de apalancarse a hacia la educación superior. Ciertamente, los maestros reconocieron que en el sector existen diferentes oportunidades para acceder a estudios universitarios, 
pero los estudiantes y sus familias no las contemplan sencillamente porque no poseen esa aspiración. Este es un ángulo que problematiza aún más la restringida orientación propedéutica para la educación superior que, según Weiss (2017), continúa priorizando el plan de estudio del telebachillerato.

\section{Desempeño académico bajo}

Otro de los problemas educativos que se hace patente en Tierras Negras tiene que ver con el bajo desempeño académico que presentan los estudiantes del sector. Existe consenso entre los diferentes maestros respecto a las enormes dificultades que supone para ellos adelantar un proceso educativo exitoso con estudiantes que presentan serias deficiencias incluso en aspectos como lectura, escritura y operaciones matemáticas básicas. Refiriéndose a sus estudiantes, un maestro analiza: no saben leer, no comprenden mucho, en cuanto a las operaciones básicas, no saben, esa es la principal problemática. Este planteamiento armoniza con lo hallado por Guzmán (2018) en un estudio adelantado en telebachilleratos comunitarios de Morelos y Yucatán.

De acuerdo con la perspectiva de los mismos maestros, son tres las circunstancias que han provocado este fenómeno: en primer lugar, los vacíos académicos que se han originado en los niveles precedentes, especialmente por las insuficiencias que presenta el aula multigrado y la ausencia de cualificación de los profesores de CONAFE; en segundo lugar, y como uno de los argumentos más marcados, la escasa articulación entre los diferentes niveles (primaria, secundaria y bachillerato); según lo entienden, el alto flujo de profesores que se ha promovido en los últimos años ha impedido que los docentes puedan formar relaciones laborales de mayor afinidad para la realización de proyectos transversales, por ejemplo: establecer la trazabilidad del proceso de cada estudiante en su paso por los diferentes niveles.

En la misma línea, los maestros dejan entrever que la falta de articulación entre los diferentes niveles también obedece a los mar- 
cados límites entre los programas que los ofrecen, en particular, cuestionan la gestión de la Secretaría de Educación toda vez que no se ha interesado por garantizar la unidad en las instituciones. De hecho, llama la atención que en el centro educativo de Tierras Negras existan claras fronteras -físicas y administrativas- entre la primaria, la secundaria y el bachillerato, por ejemplo: el restaurante escolar presta sus servicios exclusivamente a un único nivel. En este sentido uno de los maestros analiza:
A lo mejor una de las cosas que dificulta la propia arti- culación es que en la Secretaría lo vean como un solo programa de educación básica, que no lo vean preesco- lar una cosa, primaria una, secundaria otra y preparatoria otra. Si hoy la educación básica está considerada desde el preescolar hasta la preparatoria entonces se tiene que ver como un todo, y la propia Secretaría tendría entonces que generar dentro de sus programas de fortalecimiento la articulación entre los diferentes niveles.

Aunado a lo anterior, y como tercera circunstancia, los maestros reconocen que la precariedad de recursos pedagógicos con que cuenta la institución ha impedido que ellos puedan ofrecer un servicio educativo de mejor calidad, lo que repercute directamente en la motivación así como en el desempeño de los mismos estudiantes, situación que contraviene lo encontrado por Guzmán (2018) en un telebachillerato comunitario de Veracruz, donde los estudiantes expresan mayor motivación por actividades variadas y dinámicas que se realizan fuera del salón de clases.

Según lo conciben los maestros, la ausencia de espacios y de material específico para cada disciplina ha conducido a que todas las asignaturas que conforman el plan de estudios se enseñen de la misma forma, menoscabando de esta manera las didácticas que les son propias a cada una. Ciertamente, en el centro educativo de Tierras Negras existe una única aula para cada nivel (una para secundaria y una para bachillerato) dentro de la cual se deben impartir todas las materias; no hay centro de cómputo, no hay laboratorios de ciencias, no hay salón de artes, entre otros espacios que son 
necesario para ofrecer una educación integral y de mejor calidad. Este parece ser el panorama de planteles análogos en otros estados: "la falta de laboratorios y bibliotecas, con lo más que cuentan los planteles es con un pequeño acervo de diccionarios, libros de texto y algunos libros de literatura donados" (Guzmán, 2018: 86).

\section{Selección y nombramiento de docentes}

Uno de los problemas educativos más marcados en el Polígono de Tierras Negras tiene que ver con las deficiencias en el proceso de selección y nombramiento de docentes. Según la óptica de los maestros participantes, existe cierta reticencia por parte de los licenciados en pedagogía a aceptar una plaza de trabajo en el medio rural debido a las condiciones de trabajo, lo cual se ha sopesado favoreciendo la contratación de profesionistas de otras disciplinas que si bien no cuentan con formación pedagógica de base sí tienen la disponibilidad para fungir como enseñantes. El problema del nombramiento estriba en que algunos docentes están contratados temporalmente, esto quiere decir que prestan sus servicios durante una temporada y luego son trasladados, abriendo de esta manera un periodo de incertidumbre entre la comunidad educativa en general respecto a la contratación o no de un nuevo profesional. Así lo entiende uno de los maestros:

Lo que nos ha golpeado al videobachillerato es la movilidad de personal de profesores, yo creo que sí ha afectado a la matrícula; hay algunos profesores que son interinos, solo terminan el semestre, tenemos la incertidumbre si va a continuar o nos van a mandar a otra persona, tenemos dos profesores que son interinos, probablemente tengo incertidumbre: están trabajando muy bien, hemos hecho la requisición de haber si nos los pueden dejar, yo creo que sí.

Las consecuencias a las que este hecho conlleva son, por una parte, el descredito de la institución educativa frente a las familias del sector, lo que puede repercutir en la falta de credibilidad, así 
como en la disminución de la matrícula y, por otra, la imposibilidad de asegurar proyectos educativos que maduren en el tiempo. Según lo reportan los maestros, cada vez que llega un nuevo docente se ven impelidos a incorporar ciertas modificaciones al esquema ya establecido, lo que inevitablemente entorpece la dinámica de trabajo que se había constituido hasta el momento.

En lo relativo a los procesos de selección, el problema central radica en la discontinuidad de los perfiles. De acuerdo con la descripción de los maestros, la instancia encargada de la contratación de docentes solicita a los profesionales del centro un informe sobre los perfiles que requieren, incluso ellos mismos pueden postular a colegas cercanos, sin embargo, es esa instancia quien en definitiva selecciona y contrata al docente. El problema, según ellos, es que frecuentemente los docentes contratados no corresponden con los perfiles solicitados, provocando serias dificultades para la organización de la carga académica y la orientación de todas las asignaturas con la mayor idoneidad. Este hecho coincide con la percepción de maestros en el estado de México, quienes manifestaron que "la coordinación de TBC parecía una "agencia de empleos" porque contrataba a muchos recomendados e influyentes" (Weiss, 2017: 39).

Al respecto, uno de los maestros analiza:

Como ya tenemos un poco especializadas las materias digamos el profesor que da las materias de química, viene el que es especialista de química, no vamos a dejar a un licenciado (en pedagogía) que de química al menos que sea un licenciado en matemáticas, pero un licenciado en derecho o algo si, no vamos a dejarle las matemáticas al menos de que no tengamos otra persona. El SABES es quien selecciona a los docentes, a nosotros solo nos solicitan información ¿Qué requieres? ¿Qué perfil refieres? Y si tenemos algunos conocidos profesores por acá mandamos el currículum, para ver si los contratan, pero todo lo determinan en la oficina central. 
En Tierras Negras, de los seis docentes que laboran en la secundaria y el bachillerato, cuatro son ingenieros de base y tan sólo dos son licenciados en pedagogía. No es difícil imaginar, entonces, las dificultades que supone para ellos definir quién se hará cargo de las asignaturas humanísticas y de los desafíos que tal empresa entraña para quienes resulten designados. "Tenemos todas las asignaturas que nos gustan o tal vez no nos gustan, pero tenemos que darlas, otras a lo mejor ni las conozco, pero tengo que investigar para saber, eso hace parte de la telesecundaria y así tenemos que trabajar", alude uno de los maestros. De hecho, ellos mismos reconocieron que, en algunas asignaturas para las cuales no cuentan con ningún tipo de preparación, el desarrollo de éstas se convierte en un auténtico proceso de aprendizaje. Este planteamiento armoniza con Guzmán (2018: 119) cuando problematiza que "las dificultades para la elaboración de las secuencias didácticas se agravan debido a que los docentes que las imparten por primera vez no tienen el perfil profesional para impartirlas o están a cargo de demasiadas asignaturas".

\section{Los roles del docente y del estudiante}

Según lo conciben los maestros, la principal ventaja del trabajo que ellos desarrollan en Tierras Negras estriba en que pueden construir alteridades de mayor confianza y empatía con los estudiantes que tienen a su cargo. Esto obedece a que la matrícula no es muy alta (12 estudiantes por aula en promedio) y a que pueden compartir con el mismo grupo la mayor parte de la jornada todos los días de la semana. Ciertamente, reconocen que esto les ha permitido penetrar en la vida personal de sus estudiantes para conocer mucho más de cerca su ambiente sociofamiliar, todo lo cual ha contribuido a diagnosticar de mejor modo a cada uno de ellos y por esta vía diseñar acciones de acompañamiento más pertinentes. Este es un aspecto favorable del programa que, con ciertos matices, también describen Weiss (2017) y Guzmán (2018) en sus respectivos estudios. Al respecto, uno de los maestros arguye: 


\begin{abstract}
Al ser grupos de una matrícula baja, obviamente que nos permite conocer de una mejor manera al alumno, su contexto familiar y eso al final de cuentas nos da una idea sobre por qué el alumno tiene un bajo nivel, porque hay ocasiones en la que llegan: "no quiero hacer eso, porque tengo que hacerlo". De alguna manera nos sirve el conocer el contexto familiar de los alumnos, para implementar estrategias que nos permitan ayudar a los alumnos en su desempeño académico.
\end{abstract}

Igualmente, los maestros ponen de relieve que estas mismas circunstancias han favorecido la construcción de vínculos de confianza y reciprocidad con los padres de familia y con la comunidad en general, constituyéndose en un factor determinante para que las familias continúen enviando a sus hijos a la institución. Adicionalmente, analizan cómo la proximidad que se puede establecer con los padres de familia redunda en que éstos incrementen su sentido de pertenencia por el centro educativo y por esta vía se encuentren mucho más dispuestos a participar de sus procesos, especialmente en lo que se refiere a solventar algunas necesidades materiales que demandan su contribución.

Con todo, también reconocen que en el interior del aula se han sentido limitados para operacionalizar los roles que les son propios a los dos actores del proceso: docente facilitador y estudiante autónomo. Según lo describen, tanto la telesecundaria como el telebachillerato, estilan una metodología de aprendizaje que comporta la observación de videos educativos y el trabajo independiente con los libros de textos, en cuyos términos ellos fungen exclusivamente como guías, así se encuentra consignado en los documentos de la Subsecretaría de Educación Media Superior: "la figura del docente es entendida como un facilitador que organiza ambientes de aprendizaje a partir de los cuales los estudiantes pueden estructu $\neg$ rar nuevos saberes y desarrollar habilidades" (SEMS, 2015: 14). Sin embargo, dicho ideal se frustra en la práctica concreta toda vez que los estudiantes adolecen de las facultades cognitivas que se requieren para asumir por cuenta propia el alto grado de autonomía que demanda este tipo de modelos. 
Según la perspectiva de los maestros, son dos las circunstancias que han conducido a este fenómeno: en primer lugar, y como ya se ha hecho notar, los estudiantes presentan un rezago académico sustancial en comprensión de lectura y operaciones matemáticas básicas, de modo que muchos de ellos carecen de las competencias mínimas para el grado que cursan, lo que se traduce en serias dificultades para entender por cuenta propia los contenidos que se exponen en los videos y en los mismos libros de texto. "Hay libros que tiene el contenido muy bien como inglés, pero como viene diseñado para las capacidades y habilidades de ese momento, mis alumnos no van a poder, entonces tengo que entrar más como la educación tradicional de enseñarles", problematiza uno de los maestros.

En segundo lugar, los maestros ponen el acento en una suerte de discontinuidad entre las metodologías que se implementan en la primaria y las que se priorizan en la secundaria y el bachillerato, según lo entienden, en la primaria hay una alta cuota de exposición por parte del docente así como un acompañamiento diferenciado según el ritmo de aprendizaje de cada estudiante, mientras que en la etapa subsiguiente se reduce casi por completo la explicación directa por parte del docente a la vez que los apoyos pedagógicos (video y libros) están diseñados para un estudiante promedio. Así lo describe uno de los maestros:

En la primaria tienen una manera diferente de trabajar que ellos son más dedicados a lo que es la exposición, al trabajo; trabajan de manera diferente a nosotros y hemos tenido problemas precisamente al acondicionamiento del alumno, de la manera de trabajar de secundaria, todavía viene y quieren que los agarremos y los llevemos de la mano y los guiemos en el trabajo, si no lo hacemos así he notado que los niños empiezan a bajar su rendimiento, entonces en determinado momento si hemos dejado a un lado la televisión de apoyo de señal, bueno al menos yo lo he hecho así.

En lo fundamental, los profesores que trabajan en Tierras Negras presentan serias dificultades para orientar su práctica según 
los criterios pedagógicos definidos por la telesecundaria y el telebachillerato. No es un detalle menor que algunos de ellos suspendan en repetidas oportunidades los programas televisivos y el uso de los mismos libros de texto, para enfocarse en una clase expositiva (propia de una pedagogía convencional) para la cual no todos cuentan con la suficiente preparación. Finalmente, también subrayaron que los planes de estudios y los mismos procesos de evaluación y promoción son tan rígidos que frecuentemente se vean impelidos a transitar por el temario establecido sin la certeza de que sus estudiantes realmente están aprendiendo, lo cual corresponde con el planteamiento de Guzmán (2018) cuando problematiza la inadecuación de los planes de estudio a las condiciones académicas y sociales de los estudiantes, así como las limitaciones de tiempo con que cuentan los maestros para conseguir que sus dirigidos puedan alcanzar los objetivos programados en cada asignatura.

\section{Idoneidad y formación docente}

Profundizando en el tema del nombramiento y la selección de los docentes, también emergieron planteamientos sobre la idoneidad, pedagógica y disciplinar, de éstos para el ejercicio de sus funciones en el interior del aula. De hecho, los mismos maestros son críticos respecto a las innumerables exigencias que comporta trabajar en Tierras Negras y las no pocas limitaciones que experimentan para ello. Se les exige enseñar todas las asignaturas del plan de estudios, incluso aquellas para las cuales no cuentan con ningún tipo de conocimientos, se supone que esto se mitiga bajo el argumento de que no necesariamente deben dominar contenidos disciplinares, los cuales vienen definidos en los videos y los libros de texto, sino fungir como facilitadores del proceso, no obstante, y como ya se hizo notar, esto frecuente no puede operacionalizare en la práctica concreta. En este sentido, uno de los maestros comenta:

Yo recuerdo cuando llegue al videobachillerato de docente, me toco dar 11 materias, soy ingeniero agrónomo y me toco dar una materia de artes, de artes no sé nada, 
la tenía que dar y me sirvió para aprender, pero yo estoy para enseñar a los muchachos... yo aprendí mucho porque me tuve que preparar para poderlas dar.

Si bien los maestros enuncian que impartir una asignatura desmarcada de su dominio disciplinar supone para ellos un desafío de saber que los induce a prepararse, en la práctica esta situación obstruye la lógica de los procesos de enseñanza-aprendizaje y termina por lesionar el desempeño académico de los mismos estudiantes. Es cierto, tanto la telesecundaria como el telebachillerato, no demandan un cuerpo docente formado en todas las disciplinas, sino un reducido número de profesionistas que puedan fungir como facilitadores del proceso haciendo uso de los recursos audiovisuales y escritos en los que vienen definidos los contenidos para cada asignatura, pero como ya se explicó, esto no opera en la práctica concreta.

Ahora, otro factor que está obstruyendo los ideales del programa y que pone en vilo la idoneidad de los maestros, consiste en el carácter marcadamente práctico de los libros de texto y la ausencia de la fundamentación teórica que éstos deberían privilegiar. Según lo juzgan los maestros, las últimas versiones de los libros de texto suministrados por la Secretaría de Educación se han enfocado más en los ejercicios prácticos que deben desarrollar los estudiantes que en la exposición de los marcos teóricos que fundamentan cada una de las temáticas. Esto, sumado a que no existen bibliotecas cercanas y a que la conectividad a internet es prácticamente inexistente, ha conducido a que los mismos profesores tengan que preparar y exponer esa fundamentación por cuenta propia. Este hecho llama particularmente la atención toda vez que se contrapone con lo hallado por Weiss (2017) y Guzmán (2018), en cuyos planteamientos se valoran los libros de texto como un material de apoyo bien estructurado e imprescindible para orientar a los docentes profesionistas recién contratados, a tal grado que incluso son utilizados por maestros experimentados en otras modalidades de bachillerato.

Así pues, se les exige a los docentes fungir como facilitadores (cuando los estudiantes no tienen como asumir el protagonis- 
mo de su propio proceso), apoyarse en los recursos pedagógicos (cuando los libros de texto carecen de la fundamentación teórica para el desarrollo de los temas) y conducir los diferentes ritmos de aprendizaje de suerte que el grupo avance en bloque (cuando hay grandes disparidades y un programa inflexible por cumplir). Por todo lo anterior, juzgan ellos, sentirse expuestos a una diversificación de funciones que desbordan sus competencias, las cuales, a su vez, presentas serios obstáculos que hacen más espinoso su desarrollo en la práctica cotidiana. Así lo problematiza uno de los maestros:

\begin{abstract}
Realmente eso es lo que nos piden, donde veamos todos los ritmos de aprendizaje, los diferentes modelos, que atendamos al que tiene rezago y que atendamos al que va muy bien, que impartamos todas las asignaturas, y si vemos todo y todo va a depender de nosotros, como van a trabajar los alumnos, cómo aprenden, pues esa es tu planeación si lo estás llevando a cabo o no, sin importar muchas cosas que estamos viendo ahora en nuestro alrededor.
\end{abstract}

Un elemento que, en primera instancia, podría operar como contrapeso a todos estos desafíos, es la formación permanente del profesorado, sin embargo, se pudo conocer que ésta no logra surtir los efectos deseados. De hecho, existe consenso entre los maestros respecto a que la formación que reciben presenta un cierto halo de idealización de la práctica pedagógica que no se corresponde con el conjunto de circunstancias particulares que ellos enfrentan en la escuela. Los dos testimonios que se exponen a continuación ilustran este planteamiento:

Nosotros hemos ido a cursos y talleres que nos imparten y me da mucha risa, me pongo a pensar bueno a quién le hago caso, bueno cada quien tiene sus razones, pero, por ejemplo, vemos el de matemáticas y nos dicen "dale su espacio, dale tiempo", y yo no más tengo 50 minutos para trabajar en actividades; yo sé que es importante darle su tiempo y que los niños generen, y a qué hora voy a 
trabajar las otras asignaturas y es que así nos traen... todo lo que nosotros quisiéramos que el niño experimentara, viera y buscara, pero no tenemos el tiempo para hacerlo.

Al final de cuentas que esas investigaciones son de universidades privadas, entonces yo cómo puedo bajar todo ese material a un lugar que para empezar no tenemos las condiciones del medio, ni económicos, ni tecnológicos. Aquí yo que puedo hacer como maestro de una zona rural de alta marginación para implementar aquí dentro de mi plantel, ven conmigo entérate lo que pasa en mi plantel y entonces en base a eso se plantea una propuesta, que es lo que yo puedo hacer.

Lo anterior está indicando que la formación ofrecida a los profesores que ejercen en el Polígono de Tierras Negras parte de unos supuestos abstractos sobre la práctica pedagógica, emanados de planteamientos exclusivamente teóricos, que no logran responder a los lugares de emergencia que experimentan los maestros en la cotidianidad de la escuela. Este hecho también es cuestionado por Weiss et al. (2017) a propósito de las aportaciones reales que este tipo de formación proporciona a los docentes para enfrentar los desafíos que se les presentan con los estudiantes en el aula. Según los maestros, mucho más pertinentes han resultado los consejos técnicos escolares y otras estrategias afines, en las que se privilegia el encuentro entre maestros, incluso de diferentes sedes, para socializar sus dificultades y compartir sus experiencias. De hecho, Weiss (2017) subraya la importancia de generar espacios colegiados a nivel de zona o región como mecanismo para mitigar el aislamiento de los docentes y por esta vía garantizar mayores niveles de acompañamiento en el mejoramiento de la práctica docente.

\section{Conclusiones}

La telesecundaria y el telebachillerato comunitario se han erigido como dos programas educativos diferenciados para la población 
rural y urbano marginal en México, que han contribuido a cerrar las brechas de cobertura y de esta manera a incrementar el índice de jóvenes que pueden completar su ciclo de educación básica y media en estos sectores. No obstante, los resultados de pruebas nacionales e internacionales, así como los hallazgos de algunos estudios cualitativos llevados a cabo en diferentes Estados, han dejado entrever las debilidades de los dos programas en orden a ofrecer una educación de calidad.

Lo encontrado en el Polígono de Tierras Negras guarda cierto grado de continuidad con lo que reposa en la literatura, no obstante, también pone sobre la mesa un conjunto de hallazgos particulares que pueden aportar a la discusión y toma de decisiones por parte de las autoridades educativas del Estado de Guanajuato, a la vez que ofrece otras perspectivas para continuar la reflexión sobre algunos temas transversales que configuran el campo de saber formado alrededor de estos programas. En concreto, se considera necesario y pertinente mantener activa la reflexión sobre los siguientes aspectos:

La contribución de los dos programas al afianzamiento de un proyecto de vida autodeterminado por parte de los jóvenes y en este sentido el aporte real al desarrollo comunitario; el papel que cumplen los programas de becas en la forma como las familias y los mismos estudiantes le confieren sentido a la educación recibida; la articulación de los dos programas con la disposición y las oportunidades de acceso a la educación superior; el rezago académico de los estudiantes producto de la educación recibida en los niveles precedentes y las dificultades que esto supone para la operacionalización de los principios pedagógicos de los dos programas; la marcada desarticulación pedagógica y administrativa entre los programas que ofrecen la primaria, la secundaria y la educación media superior; la ausencia de espacios escolares diferentes al aula regular para ofrecer una educación integral y de mejor calidad; la contratación de docentes para cubrir con la mayor idoneidad las diferentes áreas que conforman el plan de estudios; los obstáculos de diferente naturaleza que obstruyen la posibilidad de que los docentes puedan actuar como auténticos facilitadores y guías; el 
volumen de ejercicios prácticos que privilegian los libros de texto en detrimento de la fundamentación teórica de las diferentes temáticas; y la discontinuidad que se presenta entre la formación que reciben los docentes y las dificultades reales que tienen que enfrentar en la cotidianidad de la vida escolar.

\section{Referencias bibliográficas}

Calixto, R. \& Rebollar, A. (2008). La Telesecundaria ante la sociedad del conocimiento. Revista Iberoamericana de Educación, 44(7), 1-11.

Guzmán, C (2018). Avances y dificultades en la implementación del marco curricular común. Telebachillerato estatal, educación media superior a distancia y telebachillerato comunitario. México: INEE.

Instituto Nacional de Evaluación de la Educación. [INEE]. (2019). Panorama educativo de México. Indicadores del Sistema Educativo Nacional, 2018. México: Autor.

Jiménez, J., Martínez, R. \& García, C. (2010). La Telesecundaria en México. Un breve recorrido histórico por sus datos y relatos. México: Secretaría de Educación Pública.

Salazar, P. (2007). Un estudio de estrategias y prácticas de los docentes en relación con las matemáticas de los profesores del telebachillerato en el estado de Veracruz. Tesis inédita de Maestría. México: Instituto Politécnico Nacional.

Santos del Real, A. \& Carvajal, E. (2001). Operación de las Telesecundarias en zonas rurales marginadas de México. Revista Latinoamericana de Estudios Educativos, 31(2), 69-96.

Secretaria de Educación Pública. [SEP]. (2011). Modelo educativo para el fortalecimiento de telesecundaria. Documento base. México: Autor.

SEDESOL. (2013). Catálogo de Localidades, Sistema de Apoyo para la Planeación del PDZP. México: Autor. 
Subsecretaría de Educación Media Superior. [SEMS]. (2015). Documento base. Telebachillerato Comunitario. México: Autor.

Weiss, E. (2017). Estudio exploratorio del modelo de telebachillerato comunitario y su operación en los estados. México: INEE.

Weiss, E., Antonio, L., Bernal, E., Guzmán, C. y Pedroza, P. (2017). El Telebachillerato comunitario. Una innovación curricular a discusión. Revista Latinoamericana de estudios educativos, 47(3-4), 7-26. 